MATHEMATICS OF COMPUTATION

Volume 74, Number 252, Pages 1743-1758

S 0025-5718(05)01762-X

Article electronically published on June 7, 2005

\title{
A PARAMETER ROBUST NUMERICAL METHOD FOR A TWO DIMENSIONAL REACTION-DIFFUSION PROBLEM
}

\author{
C. CLAVERO, J. L. GRACIA, AND E. O'RIORDAN
}

\begin{abstract}
In this paper a singularly perturbed reaction-diffusion partial differential equation in two space dimensions is examined. By means of an appropriate decomposition, we describe the asymptotic behaviour of the solution of problems of this kind. A central finite difference scheme is constructed for this problem which involves an appropriate Shishkin mesh. We prove that the numerical approximations are almost second order uniformly convergent (in the maximum norm) with respect to the singular perturbation parameter. Some numerical experiments are given that illustrate in practice the theoretical order of convergence established for the numerical method.
\end{abstract}

\section{INTRODUCTION}

In this work we consider the two dimensional Dirichlet boundary value reactiondiffusion problem

$$
L u=f,(x, y) \in \Omega, \quad u=g,(x, y) \in \partial \Omega,
$$

where the differential operator is defined by

$$
L \omega=-\varepsilon\left(\omega_{x x}+\omega_{y y}\right)+b(x, y) \omega .
$$

The diffusion parameter satisfies $0<\varepsilon \leq 1$ and it can be arbitrarily small. The domain $\Omega=(0,1)^{2}$ is the unit square, the reaction term satisfies $b(x, y) \geq 2 \beta>0$, and we assume that $f, b \in C^{4, \alpha}(\bar{\Omega})$ and $g \in C^{4, \alpha}(\partial \Omega)$. We also assume that there are sufficient compatibility conditions (see [4]) in order that $u \in C^{4, \alpha}(\bar{\Omega})$. This type of problem is characterized by the presence of a regular exponential layer in a neighbourhood of $\partial \Omega$ of width $O(\sqrt{\varepsilon})$. It is well known [3] that uniform meshes are an inappropriate discretization of the domain when these boundary layers are present in the solution.

It is known that the differential operator $L$ satisfies a comparison principle: For any $y \in C^{2}(\Omega)$, if $y \geq 0$ on $\partial \Omega$ and $L y \geq 0$ in $\Omega$, then $y \geq 0$ for all points in the closed domain $\bar{\Omega}$.

To simplify the notation, we consider the following subsets of the boundary $\partial \Omega$ :

$$
\begin{array}{ll}
\Gamma_{1}=\{(x, 0) \mid 0 \leq x \leq 1\}, & \Gamma_{2}=\{(0, y) \mid 0 \leq y \leq 1\} \\
\Gamma_{3}=\{(x, 1) \mid 0 \leq x \leq 1\}, & \Gamma_{4}=\{(1, y) \mid 0 \leq y \leq 1\}
\end{array}
$$

Received by the editor May 19, 2004.

2000 Mathematics Subject Classification. Primary 65N06, 65N12, 65N15; Secondary 35J25.

Key words and phrases. Reaction-diffusion, uniform convergence, Shihskin mesh, second order.

This research was partially supported by the Diputación General de Aragón and the project MCYT/FEDER BFM2001-2521. 
which are the edges of $\partial \Omega$, and the four corners of the domain are denoted by

$$
c_{1}=\Gamma_{1} \cap \Gamma_{2}, \quad c_{2}=\Gamma_{2} \cap \Gamma_{3}, \quad c_{3}=\Gamma_{3} \cap \Gamma_{4}, \quad c_{4}=\Gamma_{4} \cap \Gamma_{1} .
$$

We adopt the following notation for the boundary conditions:

$$
g(x, y)=g_{i}(x),(x, y) \in \Gamma_{i}, i=1,3 ; \quad g(x, y)=g_{i}(y),(x, y) \in \Gamma_{i}, i=2,4 .
$$

There is an extensive literature on numerical methods for singularly perturbed reaction diffusion problems (see, for example, 11, 10, 12, 17, and the references therein). Our interest lies in examining parameter-uniform numerical methods [3, 8. for singularly perturbed problems. That is, we are interested in numerical methods for which the following error bound can be theoretically established:

$$
\left\|u-\bar{U}^{N}\right\|_{\infty} \leq C N^{-p}, \quad p>0,
$$

where $N$ is the number of mesh elements employed in each coordinate direction, $\bar{U}^{N}$ is a polynomial interpolant generated by the numerical method, $\|\cdot\|_{\infty}$ is the global pointwise maximum norm, and $C$ is a constant independent of $\varepsilon$ and $N$. In general the gradients of the solution of (1.1) become unbounded as $\varepsilon \rightarrow 0$; however, parameter-uniform numerical methods guarantee that the error in the numerical approximation is controlled solely by the size of $N$.

Li and Navon [6] studied (1.1) within a finite element framework and established a convergence result in the $L_{2}$-norm. Note that the boundary layer function $e^{-x / \sqrt{\varepsilon}}$ is of measure $O\left(\varepsilon^{0.25}\right)$ in both the $L_{2}$-norm $\|\cdot\|_{0}$ and the standard energy norm $\left\||| f||^{2}=\varepsilon\right\| f_{x}\left\|_{0}^{2}+\varepsilon\right\| f_{y}\left\|_{0}^{2}+\right\| f \|_{0}^{2}$. Hence, the size of the boundary layer function is negligible in these norms. In this paper, we establish a convergence result in the pointwise norm $\|\cdot\|_{\infty}$, where $\left\|e^{-x / \sqrt{\varepsilon}}\right\|_{\infty}=1$.

In the original work of Shishkin [13, parameter-uniform numerical methods were established for an extensive class of linear singularly perturbed differential equations in $n$-dimensions, including convection-diffusion $(-\varepsilon \Delta u+\vec{a} \cdot \nabla u+b u, \vec{a}>\overrightarrow{0}, b \geq 0)$ and reaction-diffusion $(-\varepsilon \Delta u+b u, b>0)$. In [13, the author demonstrated the extent of the class of singularly perturbed problems for which the method (simple finite difference operator combined with an appropriate tensor product of piecewiseuniform meshes) could be applied, by assuming minimal regularity on the data. As in the case of nonsingularly perturbed problems, it is possible to obtain parameteruniform numerical methods with a higher order of parameter-uniform convergence by restricting the class of problems to problems with smoother data.

In one dimension, standard finite difference operators on an appropriate layeradapted mesh (Shishkin type, Bakhvalov, or variants [7, 8, 11]) yield, up to a possible logarithmic factor, parameter-uniform first order convergence for convectiondiffusion problems $\left(-\varepsilon u^{\prime \prime}+a u^{\prime}+b u, a>0, b \geq 0\right)$. For the one dimensional singularly perturbed reaction-diffusion problem $\left(-\varepsilon u^{\prime \prime}+b u, b>0\right)$ parameter-uniform second order, again up to a possible logarithmic factor, is possible 9, 15. In the case of convection-diffusion problems in two space dimensions, first order schemes have been examined by several authors (for example, see [8, 13]). In this paper we present a parameter-uniform second order scheme for the reaction-diffusion problem in two space dimensions.

The analytic properties of the solution to the two dimensional reaction-diffusion problem have been studied by [2] and [4. In this paper, we take the approach of Shishkin [13 to establish parameter-explicit a priori bounds on the derivatives of 
the solutions, which are central in deriving our theoretical asymptotic error bound on the numerical approximations.

The paper is structured as follows. In Section 2 we give the bounds of the exact solution and its derivatives showing their asymptotic behaviour with respect to the singular perturbation parameter. Moreover, we give an appropriate decomposition of this exact solution. In Section 3 we analyze the standard central finite difference scheme constructed on a special mesh of Shishkin type, proving its $\varepsilon$-uniform convergence of second order. Finally, in Section 4 we show some numerical results, which illustrate the analytical results previously proved.

Notation. Throughout this paper, $C$ denotes a generic positive constant which is independent of the diffusion parameter $\varepsilon$ and the discretization parameter $N$. We also use the following notation for the partial derivatives:

$$
f^{(k, j)}=\frac{\partial^{k+j} f}{\partial x^{k} \partial y^{j}} .
$$

\section{Decomposition And a PRIORI Bounds}

In this section we examine the asymptotic behaviour of the solution of (1.1) with respect to the singular perturbation parameter $\varepsilon$. This behaviour will be used later in the analysis of the uniform convergence of the finite difference approximations defined in Section 3.

From Han and Kellogg [4] we have that if $f, b \in C^{2, \alpha}(\bar{\Omega}), g_{s} \in C^{4, \alpha}([0,1]), s=$ $1,2,3,4$, and compatibility conditions of second level are satisfied at each of the four corners, that is,

$$
\begin{aligned}
& g_{1}(0)=g_{2}(0), \\
& -\varepsilon g_{1}^{\prime \prime}(0)-\varepsilon g_{2}^{\prime \prime}(0)+b(0,0) g_{1}(0)=f(0,0), \\
& \varepsilon g_{1}^{(4)}(0)-\varepsilon g_{2}^{(4)}(0)+f^{(2,0)}(0,0)-f^{(0,2)}(0,0) \\
& \quad-b(0,0) g_{1}^{\prime \prime}(0)-2 b^{(1,0)}(0,0) g_{1}^{\prime}(0)-b^{(2,0)}(0,0) g_{1}(0) \\
& \quad+b(0,0) g_{2}^{\prime \prime}(0)+2 b^{(0,1)}(0,0) g_{2}^{\prime}(0)+b^{(0,2)}(0,0) g_{2}(0)=0,
\end{aligned}
$$

and similarly for the other corners, then $u \in C^{4, \alpha}(\bar{\Omega})$.

Using a stretching argument and classical results from [5, 16], we can establish crude bounds on the derivatives of the solution of the form

$$
\left\|u^{(k, j)}\right\| \leq C \varepsilon^{-k / 2-j / 2}, \quad 0 \leq k+j \leq 4,
$$

where $\|\cdot\|$ is the maximum norm.

Bounds (2.2) are not sufficient to analyze the uniform convergence of the numerical scheme studied in this paper because they do not explicitly show the presence of boundary layers in $\partial \Omega$. Below we present a decomposition of $u$ and appropriate bounds of its derivatives with respect to $\varepsilon$, which will be used in the error analysis in Section 3. This decomposition was first established by Shishkin [13], where the idea of extending the domain was introduced so that a decomposition of the solution into regular and singular components could be effected without imposing additional artificial compatibility conditions on the data. For the sake of completeness, we present a detailed derivation of these bounds here.

Let $\Omega^{*}=(-a, 1+a) \times(-a, 1+a), a>0$, be an extended domain that contains $\bar{\Omega}$ as a subset. Define smooth extensions $b^{*}, f^{*}$, and $g_{s}^{*}$ of the functions $b, f$, and $g_{s}$ 
to $\bar{\Omega}^{*}$ and $[-a, 1+a]$, respectively. Note that $\left.f^{*}\right|_{\bar{\Omega}}=f$. Let $v^{*}=v_{0}^{*}+\varepsilon v_{1}^{*}$, where $v_{0}^{*}$ is the solution of the extended reduced problem

$$
b^{*} v_{0}^{*}=f^{*}
$$

and $v_{1}^{*}$ is the solution of the problem

$$
L^{*} v_{1}^{*}=\Delta v_{0}^{*},(x, y) \in \Omega^{*}, \quad v_{1}^{*}=0,(x, y) \in \partial \Omega^{*} .
$$

Since $v_{0}^{*} \in C^{4, \alpha}\left(\bar{\Omega}^{*}\right)$, then $\triangle v_{0}^{*} \in C^{2, \alpha}\left(\bar{\Omega}^{*}\right)$. The extensions of all the functions are taken such that the compatibility conditions at the corners of $\bar{\Omega}^{*}$ of up to second order (see [4]) are satisfied. Hence $v_{1}^{*} \in C^{4, \alpha}\left(\bar{\Omega}^{*}\right)$.

The regular solution $v$ is taken to be the solution of the boundary value problem

$$
L v=f,(x, y) \in \Omega, \quad v=v^{*},(x, y) \in \partial \Omega .
$$

Applying the classical results (2.2) to the extended problem (2.3), we deduce that $\left.v^{*}\right|_{\bar{\Omega}}=v \in C^{4, \alpha}(\bar{\Omega})$ and

$$
\left\|v^{(k, j)}\right\| \leq C\left(1+\varepsilon^{1-k / 2-j / 2}\right), \quad 0 \leq k+j \leq 4 .
$$

The function $v$ is called the regular component of the solution $u$. Note that by virtue of the extension, it is not necessary to impose compatibility conditions at the corners of $\bar{\Omega}$ so that $v \in C^{4, \alpha}(\bar{\Omega})$.

Associated with the bottom edge $\Gamma_{1}$ of the domain, we have a boundary layer function $w_{1}$ that is defined as follows. The domain $\Omega$ is extended in the horizontal direction to a domain $\Omega^{* *}=(-a, 1+a) \times(0,1), a>0$. The function $w_{1}^{*}$ is the solution of

$$
\begin{array}{r}
L^{* *} w_{1}^{*}=0, \quad(x, y) \in \Omega^{* *}, \\
w_{1}^{*}=u-v, \quad(x, y) \in \Gamma_{1}, \\
w_{1}^{*}(x, 1)=0, \quad x \in[-a, 1+a], \\
w_{1}^{*}(-a, y)=w_{1}^{*}(1+a, y)=0, \quad y \in[0,1],
\end{array}
$$

and the values of the boundary conditions at the points $(x, 0)$ with $x \in(-a, 0) \cup$ $(1,1+a)$ are constructed so that $w_{1}^{*} \in C^{4, \alpha}\left(\bar{\Omega}^{* *}\right)$.

So, for $0 \leq k \leq 4$ the extensions on $(-a, 0) \cup(1,1+a)$ are constructed so that

$$
\begin{aligned}
& \left(w_{1}^{*}\right)^{(k, 0)}(0,0)=(u-v)^{(k, 0)}(0,0), \\
& \left(w_{1}^{*}\right)^{(k, 0)}(1,0)=(u-v)^{(k, 0)}(1,0), \\
& \left(w_{1}^{*}\right)^{(k, 0)}(-a, 0)=\left(w_{1}^{*}\right)^{(k, 0)}(1+a, 0)=0 .
\end{aligned}
$$

Moreover, we construct the extensions so that

$$
\left(b^{*}\right)^{(k, 0)}(-a, y)=\left(b^{*}\right)^{(k, 0)}(1+a, y)=0, \quad 0 \leq k \leq 1,
$$

for all $y \in[0,1]$.

Using the comparison principle, we deduce that

$$
\left|w_{1}^{*}(x, y)\right| \leq C \frac{(a+x)(1+a-x)}{a(1+a)} e^{-\sqrt{\beta / \varepsilon} y}, \quad(x, y) \in \bar{\Omega}^{* *} .
$$

The crude bounds (2.2) on the derivatives also apply to $w_{1}^{*}$; that is,

$$
\left\|\left(w_{1}^{*}\right)^{(k, j)}\right\| \leq C \varepsilon^{-k / 2-j / 2}, \quad 0 \leq k+j \leq 4 .
$$


We now sharpen these bounds on the derivatives in the direction orthogonal to the layer. Using (2.8) and the fact that $w_{1}^{*}(-a, y)=w_{1}^{*}(1+a, y)=0$, we get that the derivatives on the sides $x=-a$ and $x=1+a$ satisfy the bounds

$$
\left|\left(w_{1}^{*}\right)^{(1,0)}(-a, y)\right| \leq C e^{-\sqrt{\beta / \varepsilon} y} \quad \text { and } \quad\left|\left(w_{1}^{*}\right)^{(1,0)}(1+a, y)\right| \leq C e^{-\sqrt{\beta / \varepsilon} y} .
$$

On the other two sides $\left|\left(w_{1}^{*}\right)^{(1,0)}(x, 0)\right| \leq C$ and $\left(w_{1}^{*}\right)^{(1,0)}(x, 1)=0$. By differentiating the differential equation (2.6 a) w.r.t. $x$, we get that

$$
L^{* *}\left(w_{1}^{*}\right)^{(1,0)}=-\left(b^{*}\right)^{(1,0)}\left(w_{1}^{*}\right), \quad(x, y) \in \Omega^{* *},
$$

and using the comparison principle, it follows that

$$
\left|\left(w_{1}^{*}\right)^{(1,0)}(x, y)\right| \leq C e^{-\sqrt{\beta / \varepsilon} y} .
$$

Note that

$$
\left|\left(w_{1}^{*}\right)^{(2,0)}(x, 0)\right| \leq C,\left(w_{1}^{*}\right)^{(2,0)}(x, 1)=0,
$$

and from the regularity of $w_{1}^{*}$ and the fact that $\varepsilon\left(\left(w_{1}^{*}\right)^{(2,0)}+\left(w_{1}^{*}\right)^{(0,2)}\right)=b^{*} w_{1}^{*}$ in the closed region $\bar{\Omega}^{* *}$, we have that

$$
\begin{gathered}
\left(w_{1}^{*}\right)^{(2,0)}(-a, y)=\left(w_{1}^{*}\right)^{(0,2)}(-a, y)=0, \\
\left(w_{1}^{*}\right)^{(2,0)}(1+a, y)=\left(w_{1}^{*}\right)^{(0,2)}(1+a, y)=0 .
\end{gathered}
$$

Hence, after differentiating (2.6 a) twice w.r.t. $x$, the maximum principle establishes

$$
\left|\left(w_{1}^{*}\right)^{(2,0)}(x, y)\right| \leq C(a+x)(1+a-x) e^{-\sqrt{\beta / \varepsilon} y} .
$$

Using (2.11) and the fact that $\left(w_{1}^{*}\right)^{(2,0)}(-a, y)=\left(w_{1}^{*}\right)^{(2,0)}(1+a, y)=0$, we get that

$$
\left|\left(w_{1}^{*}\right)^{(3,0)}(-a, y)\right| \leq C e^{-\sqrt{\beta / \varepsilon} y} \quad \text { and } \quad\left|\left(w_{1}^{*}\right)^{(3,0)}(1+a, y)\right| \leq C e^{-\sqrt{\beta / \varepsilon} y},
$$

which yields

$$
\left|\left(w_{1}^{*}\right)^{(3,0)}(x, y)\right| \leq \frac{C}{\sqrt{\varepsilon}} e^{-\sqrt{\beta / \varepsilon} y} .
$$

Differentiating (2.6 $\mathrm{a})$ twice w.r.t. $y$ yields the fact that the mixed derivatives satisfy

$$
\left(w_{1}^{*}\right)^{(2,2)}(-a, y)=\left(w_{1}^{*}\right)^{(2,2)}(1+a, y)=0 .
$$

Using this and differentiating (2.6) twice w.r.t. $x$, it follows that

$$
\left(w_{1}^{*}\right)^{(4,0)}(-a, y)=\left(w_{1}^{*}\right)^{(4,0)}(1+a, y)=0 .
$$

Hence,

$$
\left|\left(w_{1}^{*}\right)^{(4,0)}(x, y)\right| \leq \frac{C}{\varepsilon} .
$$

Associated with the bottom edge $\Gamma_{1}$, we define a boundary layer function $w_{1}$ to be the solution of the homogeneous problem

$$
\begin{array}{r}
L w_{1}=0,(x, y) \in \Omega, \\
w_{1}=u-v,(x, y) \in \Gamma_{1}, \quad w_{1}=0,(x, y) \in \Gamma_{3}, \\
w_{1}(0, y)=w_{1}^{*}(0, y), \quad w_{1}(1, y)=w_{1}^{*}(1, y) .
\end{array}
$$

In an analogous fashion, we can define boundary layer functions $w_{k}, k=2,3,4$, associated with the three other edges, and the corresponding bounds on the derivatives of these functions will hold. 
Associated with the corner $c_{1}$, we define a corner layer function $z_{1}$ such that

$$
\begin{array}{r}
L z_{1}=0,(x, y) \in \Omega, \\
z_{1}=-w_{2},(x, y) \in \Gamma_{1}, \quad z_{1}=-w_{1},(x, y) \in \Gamma_{2}, \\
z_{1}=0,(x, y) \in \Gamma_{3}, \quad z_{1}=0,(x, y) \in \Gamma_{4} .
\end{array}
$$

Note that $L w_{1}=L w_{2}=0$ and $w_{1}, w_{2} \in C^{4, \alpha}(\bar{\Omega})$. Thus, the compatibility conditions up to second order (see [4) hold at the four corners of the domain, which implies that $z_{1} \in C^{4, \alpha}(\bar{\Omega})$. The maximum principle and the condition $b \geq 2 \beta>0$, result in the bound

$$
\left|z_{1}(x, y)\right| \leq C e^{-\sqrt{\beta / \varepsilon} x} e^{-\sqrt{\beta / \varepsilon} y} .
$$

In an analogous fashion we can define corner layer functions $z_{k}, k=2,3,4$, associated with the three other corners, and the corresponding bounds hold.

Remark 2.1. From (2.2) we have the bounds

$$
\left\|\left(w_{1}^{*}\right)^{(0,1)}\right\|,\left\|\left(z_{1}\right)^{(0,1)}\right\|,\left\|\left(z_{1}\right)^{(1,0)}\right\| \leq \frac{C}{\sqrt{\varepsilon}} .
$$

These bounds can be sharpened using the following argument. Use the barrier function

$$
\phi(x, y)=C\left(e^{-\sqrt{\beta / \varepsilon} y}-e^{-\sqrt{\beta / \varepsilon}(2-y)}\right),
$$

to get that

$$
\left|w_{1}^{*}(x, y)\right| \leq C \frac{1-y}{\sqrt{\varepsilon}} e^{-\sqrt{\beta / \varepsilon} y} \quad \text { and } \quad\left|\left(w_{1}^{*}\right)^{(0,1)}(x, 1)\right| \leq \frac{C}{\sqrt{\varepsilon}} e^{-\sqrt{\beta / \varepsilon}} .
$$

Using this and the crude bounds on the derivative of $w_{1}^{*}$, we have that

$$
\left|\left(w_{1}^{*}\right)^{(0,1)}(x, y)\right| \leq \frac{C}{\sqrt{\varepsilon}} e^{-\sqrt{\beta / \varepsilon} y} .
$$

Analogous bounds hold for $\left|\left(w_{3}^{*}\right)^{(0,1)}(x, y)\right|,\left|\left(w_{2}^{*}\right)^{(1,0)}(x, y)\right|$, and $\left|\left(w_{4}^{*}\right)^{(1,0)}(x, y)\right|$.

Now we sharpen the bounds on the first derivatives of the function $z_{1}$. From the above argument it follows that

$$
\begin{aligned}
& \left|w_{1}^{*}(x, y)\right| \leq C\left(e^{-\sqrt{\beta / \varepsilon} y}-e^{-\sqrt{\beta / \varepsilon}(2-y)}\right), \\
& \left|w_{2}^{*}(x, y)\right| \leq C\left(e^{-\sqrt{\beta / \varepsilon} x}-e^{-\sqrt{\beta / \varepsilon}(2-x)}\right) .
\end{aligned}
$$

Use the barrier function

$$
\psi(x, y)=C\left(e^{-\sqrt{\beta / \varepsilon} x}-e^{-\sqrt{\beta / \varepsilon}(2-x)}\right)\left(e^{-\sqrt{\beta / \varepsilon} y}-e^{-\sqrt{\beta / \varepsilon}(2-y)}\right),
$$

with $C$ sufficiently large and the discrete maximum principle to establish that

$$
\left|\left(z_{1}\right)^{(1,0)}(x, y)\right|,\left|\left(z_{1}\right)^{(0,1)}(x, y)\right| \leq \frac{C}{\sqrt{\varepsilon}} e^{-\sqrt{\beta / \varepsilon} x} e^{-\sqrt{\beta / \varepsilon} y} .
$$

Analogous bounds hold for the first derivatives of the other three corner layer functions.

We summarize this section with the following result: 
Theorem 2.2. The solution $u$ of (1.1) may be written as a sum

$$
u=v+\sum_{i=1}^{4} w_{i}+\sum_{i=1}^{4} z_{i},
$$

where

$$
L v=f, \quad L w_{i}=0 \quad L z_{i}=0, \quad i=1,2,3,4 .
$$

Boundary conditions for $v, w_{i}, z_{i}, i=1,2,3,4$, can be specified so that the following bounds on the derivatives of the components hold:

$$
\begin{gathered}
\left\|v^{(k, j)}\right\| \leq C\left(1+\varepsilon^{1-k / 2-j / 2}\right), \quad 0 \leq k+j \leq 4, \\
\left|w_{1}(x, y)\right| \leq C e^{-\sqrt{\beta / \varepsilon} y}, \quad\left|w_{2}(x, y)\right| \leq C e^{-\sqrt{\beta / \varepsilon} x}, \\
\left|w_{3}(x, y)\right| \leq C e^{-\sqrt{\beta / \varepsilon}(1-y)}, \quad\left|w_{4}(x, y)\right| \leq C e^{-\sqrt{\beta / \varepsilon}(1-x)}, \\
\max \left\{\left\|w_{i}^{(k, j)}\right\|,\left\|z_{i}^{(k, j)}\right\|\right\} \leq C \varepsilon^{-k / 2-j / 2}, \quad 0 \leq k+j \leq 4, \\
\left\|w_{i}^{(k, 0)}\right\| \leq C\left(1+\varepsilon^{1-k / 2}\right), \quad i=1,3, \\
\left\|w_{i}^{(0, k)}\right\| \leq C\left(1+\varepsilon^{1-k / 2}\right), \quad i=2,4,0 \leq k \leq 4, \\
\left|z_{1}(x, y)\right| \leq C e^{-\sqrt{\beta / \varepsilon} y} e^{-\sqrt{\beta / \varepsilon} x}, \\
\left|z_{2}(x, y)\right| \leq C e^{-\sqrt{\beta / \varepsilon}(1-y)} e^{-\sqrt{\beta / \varepsilon} x} \\
\left|z_{3}(x, y)\right| \leq C e^{-\sqrt{\beta / \varepsilon}(1-y)} e^{-\sqrt{\beta / \varepsilon}(1-x)} \\
\left|z_{4}(x, y)\right| \leq C e^{-\sqrt{\beta / \varepsilon} y} e^{-\sqrt{\beta / \varepsilon}(1-x)}
\end{gathered}
$$

\section{THE DISCRETE PROBLEM}

To discretize problem (1.1), we use the standard central difference operator

$$
\begin{aligned}
& L^{N} U^{N}=-\varepsilon\left(\delta_{x}^{2}+\delta_{y}^{2}\right) U+b U=f, \quad\left(x_{i}, y_{j}\right) \in \Omega^{N}, \\
& U=u, \quad \text { on the boundary } \partial \Omega^{N},
\end{aligned}
$$

where the mesh $\Omega^{N}$ is the tensor product of two one dimensional piecewise-uniform Shishkin meshes; i.e., $\Omega^{N}=\Omega_{x} \times \Omega_{y}$, where $\Omega_{x}$ (similarly for $\Omega_{y}$ ) splits the interval $[0,1]$ into three subintervals $\left[0, \sigma_{x}\right],\left[\sigma_{x}, 1-\sigma_{x}\right]$, and $\left[1-\sigma_{x}, 1\right]$. The mesh distributes $N / 4$ points uniformly within each of the subintervals $\left[0, \sigma_{x}\right]$ and $\left[1-\sigma_{x}, 1\right]$ and the remaining $N / 2$ mesh points uniformly in the interior subinterval $\left[\sigma_{x}, 1-\sigma_{x}\right]$. To simplify our discussion, we take $\sigma_{x}=\sigma_{y}$; these transition points are defined as

$$
\sigma=\sigma_{x}=\sigma_{y}=\min \left\{\frac{1}{4}, 2 \sqrt{\frac{\varepsilon}{\beta}} \ln N\right\} .
$$

Below we denote by $h=4 \sigma / N, H=2(1-2 \sigma) / N ; h_{i+1}=x_{i+1}-x_{i}, k_{i+1}=$ $y_{i+1}-y_{i}, 0 \leq i \leq N-1$, and $\bar{h}_{i}=\left(h_{i+1}+h_{i}\right) / 2, \bar{k}_{i}=\left(k_{i+1}+k_{i}\right) / 2,1 \leq i \leq N-1$.

It is well known that for the reaction-diffusion problem, central differences are an $\varepsilon$-uniformly stable scheme in the maximum norm; that is, for any mesh function $W$

$$
\|W\| \leq \frac{1}{2 \beta}\left\|L^{N} W\right\|+\max _{\partial \Omega^{N}}|W|
$$


From (3.2) we see that in order to prove uniform convergence, we only need to analyze the local truncation error. Using a standard truncation error argument, we can easily obtain

$$
\begin{aligned}
& \left|L^{N}(U-u)\left(x_{i}, y_{j}\right)\right| \\
& \leq \begin{cases}C \varepsilon\left(\bar{h}_{i}\left\|u^{(3,0)}\right\|+\bar{k}_{j}\left\|u^{(0,3)}\right\|\right), & \text { if } x_{i}=\sigma_{x}, 1-\sigma_{x}, \text { or } y_{j}=\sigma_{y}, 1-\sigma_{y}, \\
C \varepsilon\left(h_{i}^{2}\left\|u^{(4,0)}\right\|+k_{j}^{2}\left\|u^{(0,4)}\right\|\right), & \text { otherwise. }\end{cases}
\end{aligned}
$$

Nevertheless, to find appropriate bounds of this error, we need to decompose the discrete solution similarly to the decomposition of the exact solution. The numerical solution can be written in the form

$$
U=V+\sum_{k=1}^{4} W_{k}+\sum_{k=1}^{4} Z_{k}
$$

where

$$
\begin{aligned}
& \left\{\begin{array}{l}
L^{N} V=f, \text { in } \Omega^{N}, \\
V=v, \text { in } \partial \Omega^{N},
\end{array}\right. \\
& \left\{\begin{array}{l}
L^{N} W_{k}=0, \text { in } \Omega^{N}, \quad k=1,2,3,4, \\
W_{k}=w_{k}, \text { in } \partial \Omega^{N},
\end{array}\right. \\
& \left\{\begin{array}{l}
L^{N} Z_{k}=0, \text { in } \Omega^{N}, \quad k=1,2,3,4, \\
Z_{k}=z_{k}, \text { in } \partial \Omega^{N} .
\end{array}\right.
\end{aligned}
$$

From (2.5) and (3.3) a straightforward computation gives

$$
\left|L^{N}(V-v)\left(x_{i}, y_{j}\right)\right| \leq \begin{cases}C \sqrt{\varepsilon} N^{-1}, & \text { if } x_{i}=\sigma_{x}, 1-\sigma_{x}, \text { or } y_{j}=\sigma_{y}, 1-\sigma_{y}, \\ C N^{-2}, & \text { otherwise. }\end{cases}
$$

Following [9], we define the barrier function

$$
\Psi\left(x_{i}, y_{j}\right)=C \frac{\sigma_{x} \sigma_{y}}{\varepsilon} N^{-2}\left(\theta\left(x_{i}\right)+\theta\left(y_{j}\right)\right)+C N^{-2},
$$

where $\theta(z)$ is a piecewise-linear polynomial defined by

$$
\theta(z)=\left\{\begin{array}{ll}
\frac{z}{\sigma}, & 0 \leq z \leq \sigma, \\
1, & \sigma \leq z \leq 1-\sigma, \\
\frac{1-z}{\sigma}, & 1-\sigma \leq z \leq 1,
\end{array} \quad \text { and } \quad \delta_{z}^{2} \theta(z)=\left\{\begin{array}{l}
-\frac{N}{\sigma}, z=\sigma, 1-\sigma \\
0, \text { otherwise. }
\end{array}\right.\right.
$$

From the choice of transition points, it follows that

$$
0 \leq \Psi\left(x_{i}, y_{j}\right) \leq C\left(N^{-1} \ln N\right)^{2},
$$

and we also have

$L^{N}(\Psi)\left(x_{i}, y_{j}\right)= \begin{cases}C \sigma N^{-1}+(b \Psi)\left(x_{i}, y_{j}\right), & \text { if } x_{i}=\sigma_{x}, 1-\sigma_{x}, \text { or } y_{j}=\sigma_{y}, 1-\sigma_{y}, \\ (b \Psi)\left(x_{i}, y_{j}\right), & \text { otherwise }\end{cases}$

Then the discrete maximum principle gives us that

$$
\|V-v\| \leq C\left(N^{-1} \ln N\right)^{2},
$$

which is the appropriate bound for the error associated with the regular component. 
To prove $\varepsilon$-uniform bounds of the errors associated with the edge and corner functions, we use an argument based on appropriate barrier functions. As usual for singularly perturbed problems, we consider the barrier functions

$$
\begin{aligned}
& B_{w_{1} ; j}=\left\{\begin{array}{l}
\prod_{\substack{s=1 \\
1, j}}^{j}\left(1+k_{s} \sqrt{\beta / \varepsilon}\right)^{-1}, j \neq 0
\end{array}\right. \\
& B_{w_{2} ; i}=\left\{\begin{array}{l}
\prod_{s=1}^{i}\left(1+h_{s} \sqrt{\beta / \varepsilon}\right)^{-1}, i \neq 0, \\
1, i=0
\end{array}\right. \\
& B_{w_{3} ; j}=\left\{\begin{array}{l}
\prod_{\substack{s=j+1 \\
1, j=N}}^{N}\left(1+k_{s} \sqrt{\beta / \varepsilon}\right)^{-1}, j \neq N
\end{array}\right. \\
& B_{w_{4} ; i}=\left\{\begin{array}{l}
\prod_{\substack{s=i+1 \\
1, i=N .}}^{N}\left(1+h_{s} \sqrt{\beta / \varepsilon}\right)^{-1}, i \neq N,
\end{array}\right.
\end{aligned}
$$

These functions are first order Taylor approximations of the exponential functions related to the singular behaviour of the solution of problem (1.1). Note that for all $j$ it holds that

$$
\exp \left(-\sqrt{\beta / \varepsilon} y_{j}\right)=\prod_{s=1}^{j} \exp \left(-\sqrt{\beta / \varepsilon} k_{s}\right) \leq B_{w_{1} ; j},
$$

and for $\sigma<0.25$ and $N / 4 \leq j \leq N$ we have

$$
\begin{gathered}
B_{w_{1} ; j} \leq B_{w_{1} ; N / 4}=\left(1+\frac{8 \ln N}{N}\right)^{-N / 4} \leq C N^{-2}, \\
L^{N} B_{w_{1} ; j} \geq\left(b\left(x_{i}, y_{j}\right)-2 \beta\right) B_{w_{1} ; j} .
\end{gathered}
$$

Analogous bounds hold for the other three edge functions.

Proposition 3.1. If $w_{k}$ and $W_{k}$ are the solutions of (2.15) and (3.6), respectively, then, for $k=1,2,3,4$,

$$
\left|w_{k}\left(x_{i}, y_{j}\right)-W_{k}\left(x_{i}, y_{j}\right)\right| \leq C\left(N^{-1} \ln N\right)^{2}, \quad\left(x_{i}, y_{j}\right) \in \Omega^{N} .
$$

Proof. We assume throughout that $\sigma<0.25$. The case of $\sigma=0.25$ is dealt with in this case in a classical fashion by noting that $\varepsilon^{-1} \leq C(\ln N)^{2}$. We only give the details for the edge layer function $w_{1}$. The argument is analogous for the other three boundary layer functions.

From (3.6) and Theorem 2.2 it follows that

$$
\left|W_{1}\left(x_{i}, y_{j}\right)\right|=\left|w_{1}\left(x_{i}, y_{j}\right)\right| \leq C \exp \left(-\sqrt{\beta / \varepsilon} y_{j}\right) \leq C B_{w_{1} ; j}, \quad\left(x_{i}, y_{j}\right) \in \partial \Omega^{N}
$$
on the boundary $\partial \Omega^{N}$. Also for each internal mesh point $\left(x_{i}, y_{j}\right) \in \Omega^{N}, 0<i, j<$ $N$, from (3.6), (3.10), and the discrete maximum principle, it follows that

$$
\left|W_{1}\left(x_{i}, y_{j}\right)\right| \leq B_{w_{1 ; j}} .
$$

Therefore, using Theorem 2.2 and (3.13), we deduce that

$$
\left|w_{1}\left(x_{i}, y_{j}\right)-W_{1}\left(x_{i}, y_{j}\right)\right| \leq\left|w_{1}\left(x_{i}, y_{j}\right)\right|+\left|W_{1}\left(x_{i}, y_{j}\right)\right| \leq C B_{w_{1 ; j}} .
$$


Hence, for the mesh points that are not close to the edge $\Gamma_{1}$, from (3.9) we have

$$
\left|w_{1}\left(x_{i}, y_{j}\right)-W_{1}\left(x_{i}, y_{j}\right)\right| \leq C N^{-2}, \quad 0 \leq i \leq N, N / 4 \leq j \leq N .
$$

To prove similar bounds of the error in the region $\Omega_{1}^{N}=\left\{\left(x_{i}, y_{j}\right) \mid 0<i<N, 0<\right.$ $j<N / 4\}$, we proceed as follows. Using Taylor expansions, we obtain

$\left|L^{N}\left[W_{1}\left(x_{i}, y_{j}\right)-w_{1}\left(x_{i}, y_{j}\right)\right]\right| \leq \begin{cases}C \varepsilon\left(\bar{h}_{i}\left\|w_{1}^{(3,0)}\right\|+k_{j}^{2}\left\|w_{1}^{(0,4)}\right\|\right), & i=N / 4,3 N / 4, \\ C \varepsilon\left(h_{i}^{2}\left\|w_{1}^{(4,0)}\right\|+k_{j}^{2}\left\|w_{1}^{(0,4)}\right\|\right), & \text { otherwise. }\end{cases}$

From Theorem 2.2, it follows that

$$
\left|L^{N}\left[W_{1}\left(x_{i}, y_{j}\right)-w_{1}\left(x_{i}, y_{j}\right)\right]\right| \leq \begin{cases}C\left(\left(N^{-1} \ln N\right)^{2}+N^{-1} \sqrt{\varepsilon}\right), & i=N / 4,3 N / 4, \\ C\left(N^{-1} \ln N\right)^{2}, & \text { otherwise. }\end{cases}
$$

Then, similar to the analysis for the regular component, we use the barrier function

$$
\Psi\left(x_{i}, y_{j}\right)=C \frac{\sigma_{x}}{\sqrt{\varepsilon}} N^{-2} \theta\left(x_{i}\right)+C\left(N^{-1} \ln N\right)^{2}
$$

and the discrete maximum principle, now applied only on $\bar{\Omega}_{1}^{N}$, to get

$$
\left|w_{1}\left(x_{i}, y_{j}\right)-W_{1}\left(x_{i}, y_{j}\right)\right| \leq C\left(N^{-1} \ln N\right)^{2}, \quad\left(x_{i}, y_{j}\right) \in \bar{\Omega}_{1}^{N} .
$$

The result follows from (3.14) and (3.15).

Proposition 3.2. If $z_{k}$ and $Z_{k}$ are the solutions of (2.16) and (3.7), respectively, then, for $k=1,2,3,4$,

$$
\left|z_{k}\left(x_{i}, y_{j}\right)-Z_{k}\left(x_{i}, y_{j}\right)\right| \leq C\left(N^{-1} \ln N\right)^{2}, \quad\left(x_{i}, y_{j}\right) \in \Omega^{N} .
$$

Proof. Again we only give the proof of (3.16) for the corner layer function $z_{1}$ and in the case of $\sigma<0.25$. In a similar way to the argument given in Proposition 3.1. we obtain

$$
\left|Z_{1}\left(x_{i}, y_{j}\right)\right| \leq C \min \left\{B_{w_{2} ; i}, B_{w_{1} ; j}\right\}, \quad \text { if }\left(x_{i}, y_{j}\right) \in \partial \Omega^{N},
$$

and

$$
\left|z_{1}\left(x_{i}, y_{j}\right)-Z_{1}\left(x_{i}, y_{j}\right)\right| \leq C \min \left\{B_{w_{2} ; i}, B_{w_{1} ; j}\right\}, \quad \text { if }\left(x_{i}, y_{j}\right) \in \Omega^{N}, 0<i, j<N .
$$

Then, using (3.9), we deduce that

$$
\left|z_{1}\left(x_{i}, y_{j}\right)-Z_{1}\left(x_{i}, y_{j}\right)\right| \leq C N^{-2}, \quad\left(x_{i}, y_{j}\right) \in \Omega^{N} \backslash \Omega_{1,2}^{N},
$$

where $\Omega_{1,2}^{N}=\left\{\left(x_{i}, y_{j}\right) \mid 0<i, j<N / 4\right\}$.

Finally, in $\Omega_{1,2}^{N}$ the truncation error satisfies

$$
\left|L^{N}\left[Z_{1}\left(x_{i}, y_{j}\right)-z_{1}\left(x_{i}, y_{j}\right)\right]\right| \leq C \varepsilon h^{2}\left(\left\|z_{1}^{(4,0)}\right\|+\left\|z_{1}^{(0,4)}\right\|\right) \leq C\left(N^{-1} \ln N\right)^{2},
$$

where we have used Theorem 2.2. Considering the barrier function

$$
\Psi\left(x_{i}, y_{j}\right)=C\left(N^{-1} \ln N\right)^{2},
$$

the discrete maximum principle, used on $\bar{\Omega}_{1,2}^{N}$, proves the required result.

Thus, from the bounds (3.8) and Propositions 3.1 and 3.2 , we deduce the following result of uniform convergence. 
Theorem 3.3. Let $u$ be the solution of problem (1.1) and $U$ the numerical solution of (3.1) defined on the piecewise-uniform Shihskin mesh. Then the error at the mesh points satisfies

$$
\left|(u-U)\left(x_{i}, y_{j}\right)\right| \leq C\left(N^{-1} \ln N\right)^{2}, \quad\left(x_{i}, y_{j}\right) \in \Omega^{N} .
$$

A further property of Shishkin meshes is parameter-uniform interpolation. Let

$$
w_{I}(x, y)=\sum_{i, j=0}^{N} w\left(x_{i}, y_{j}\right) \phi_{i}(x) \phi^{j}(y),
$$

where $\phi_{i}(x)$ is the standard piecewise-linear basis function associated with the interval $\left[x_{i-1}, x_{i+1}\right]$. Define a global bilinear approximation to the solution $u$ of (1.1) as

$$
\bar{U}^{N}(x, y)=\sum_{i, j=0}^{N} U^{N}\left(x_{i}, y_{j}\right) \phi_{i}(x) \phi^{j}(y) .
$$

Note that from the previous theorem

$$
\left\|u-\bar{U}^{N}\right\| \leq\left\|u-u_{I}\right\|+C\left(N^{-1} \ln N\right)^{2} .
$$

From the argument in 14, the bounds on the components in the decomposition given in Theorem 2.2, and the sharper bounds on the first derivatives of the layer components given in (2.18), (2.19), we have the global parameter-uniform error bound

$$
\left\|u-\bar{U}^{N}\right\| \leq C\left(N^{-1} \ln N\right)^{2} .
$$

\section{NUMERICAL EXPERIMENTS}

In this section we present numerical results obtained by applying the numerical method described in Section 3 to two particular problems of the form (1.1). To estimate the maximum errors, we use a variant of the double mesh principle. The two mesh difference is calculated using

$$
D_{\varepsilon}^{N}=\left(\max _{0 \leq i, j \leq N}\left|\tilde{U}_{2 i, 2 j}^{2 N}-U_{i, j}^{N}\right|\right)
$$

where $\left\{\tilde{U}^{2 N}\right\}$ is the numerical solution on a mesh that contains the mesh points $\left(x_{i}, y_{j}\right)$ of $\bar{\Omega}^{N}$ and also the midpoints $x_{i+1 / 2}=\left(x_{i}+x_{i+1}\right) / 2, y_{i+1 / 2}=$ $\left(y_{i}+y_{i+1}\right) / 2, i=0,1, \ldots, N-1$. From these values we define the $\varepsilon$-uniform differences by $D^{N}=\max _{\varepsilon} D_{\varepsilon}^{N}$, the numerical orders of convergence are calculated by $p_{\varepsilon}^{N}=\log \left(D_{\varepsilon}^{N} / D_{\varepsilon}^{2 N}\right) / \log 2$, and the $\varepsilon$-uniform order is given by $p_{\text {uni }}^{N}=\log \left(D^{N} / D^{2 N}\right) / \log 2$.

The first test problem is

$$
\begin{aligned}
& -\varepsilon \Delta u+\left(1+x^{2} y^{2}\right) u=0, \quad(x, y) \in(0,1)^{2}, \\
& u(x, 0)=(1-x)^{2}, \quad 0 \leq x \leq 1, \\
& u(0, y)=1-y, \quad 0 \leq y \leq 1, \\
& u(x, 1)=u(1, y)=0, \quad 0 \leq x, y \leq 1 .
\end{aligned}
$$

Figure 1 shows the numerical solution for $\varepsilon=10^{-6}$ and $N=64$. From this figure we see that the solution has a corner layer at $(0,0)$ and two boundary layers near the edges $x=0$ and $y=0$ of the unit square. Note that the reduced solution is identically zero and that $\|u\|=1$. 


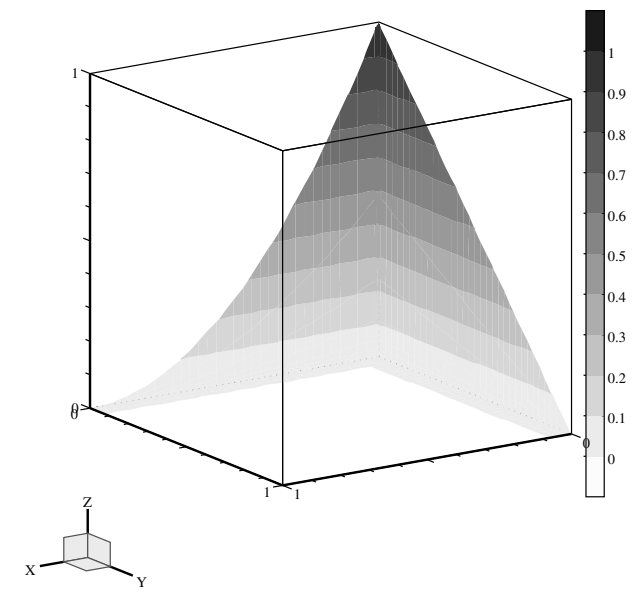

Figure 1. Numerical solution of problem (4.1) $\left(\varepsilon=10^{-6}, N=64\right)$

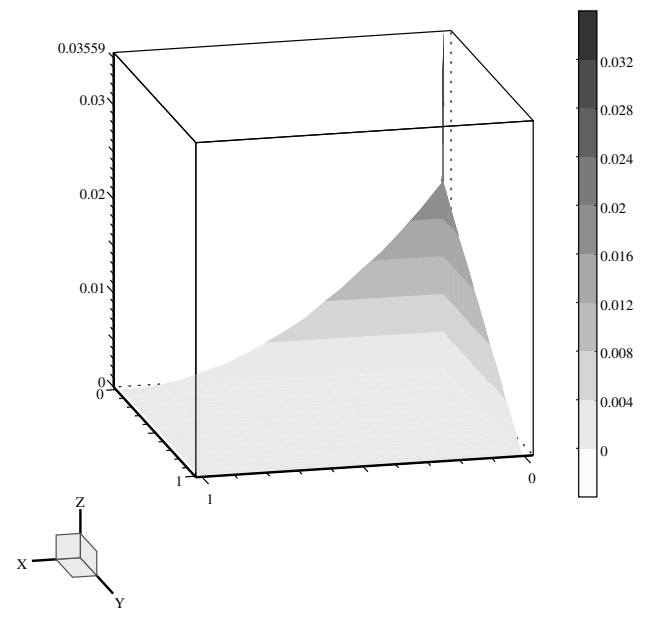

Figure 2. Approximate errors $\left\|U^{32}-\tilde{U}^{512}\right\|$ for problem (4.1) $\left(\varepsilon=10^{-6}\right)$

Table 1 displays the maximum differences $D_{\varepsilon}^{N}$ and the numerical orders of convergence $p_{\varepsilon}^{N}$ for $\varepsilon=1,2^{-2}, 2^{-4}, \ldots, 2^{-26}$. These results indicate that the uniform order of convergence is in agreement with Theorem 3.3, even though we do not have sufficient compatibility conditions (for example in the corner $(0,0)$ only the condition of order zero holds) for problem (4.1).

Figure 2 shows the approximate pointwise errors for $\varepsilon=10^{-6}$ calculated by comparing the numerical solution obtained with $N=32$ with the numerical solution obtained using a finer mesh having $N=512$, in such way that the transition points of the mesh $\Omega^{32}$ and the finer mesh $\tilde{\Omega}^{512}$ coincide. The finer mesh $\tilde{\Omega}^{512}$ is obtained by dividing each subinterval of the coarse mesh $\Omega^{32}$ into sixteen subintervals of equal length. We see that the maximum error occurs in the corner layer region. 
TABLE 1. Maximum differences $D_{\varepsilon}^{N}$ and the numerical orders of convergence $p_{\varepsilon}^{N}$ for problem (4.1)

\begin{tabular}{|c|c|c|c|c|}
\hline$\varepsilon$ & $N=32$ & $N=64$ & $N=128$ & $N=256$ \\
\hline$\varepsilon=1$ & $\begin{array}{c}3.961 E-5 \\
1.995\end{array}$ & $\begin{array}{c}9.938 E-6 \\
1.998\end{array}$ & $\begin{array}{c}2.488 E-6 \\
2.000\end{array}$ & $6.221 E-7$ \\
\hline$\varepsilon=2^{-2}$ & $\begin{array}{c}3.740 E-5 \\
1.965\end{array}$ & $\begin{array}{c}9.579 E-6 \\
1.985\end{array}$ & $\begin{array}{c}2.420 E-6 \\
1.994\end{array}$ & $6.075 E-7$ \\
\hline$\varepsilon=2^{-4}$ & $\begin{array}{c}2.651 E-4 \\
1.962\end{array}$ & $\begin{array}{c}6.804 E-5 \\
1.986\end{array}$ & $\begin{array}{c}1.718 E-5 \\
1.995\end{array}$ & $4.310 E-6$ \\
\hline$\varepsilon=2^{-6}$ & $\begin{array}{c}1.208 E-3 \\
1.945\end{array}$ & $\begin{array}{c}3.136 E-4 \\
1.984\end{array}$ & $\begin{array}{c}7.931 E-5 \\
1.995\end{array}$ & $1.989 E-5$ \\
\hline$\varepsilon=2^{-8}$ & $\begin{array}{c}4.791 E-3 \\
1.873\end{array}$ & $\begin{array}{c}1.308 E-3 \\
1.961\end{array}$ & $\begin{array}{c}3.360 E-4 \\
1.990\end{array}$ & $8.460 E-5$ \\
\hline$\varepsilon=2^{-10}$ & $\begin{array}{c}1.740 E-2 \\
1.780\end{array}$ & $\begin{array}{c}5.065 E-3 \\
1.895 \\
\end{array}$ & $\begin{array}{c}1.361 E-3 \\
1.972\end{array}$ & $3.470 E-4$ \\
\hline$\varepsilon=2^{-12}$ & $\begin{array}{c}2.472 E-2 \\
1.270\end{array}$ & $\begin{array}{c}1.025 E-2 \\
1.386 \\
\end{array}$ & $\begin{array}{c}3.922 E-3 \\
1.556\end{array}$ & $1.334 E-3$ \\
\hline$\varepsilon=2^{-14}$ & $\begin{array}{c}2.514 E-2 \\
1.281\end{array}$ & $\begin{array}{c}1.035 E-2 \\
1.384\end{array}$ & $\begin{array}{c}3.964 E-3 \\
1.559\end{array}$ & $1.345 E-3$ \\
\hline$\varepsilon=2^{-16}$ & $\begin{array}{c}2.535 E-2 \\
1.286\end{array}$ & $\begin{array}{c}1.040 E-2 \\
1.384 \\
\end{array}$ & $\begin{array}{c}3.985 E-3 \\
1.561\end{array}$ & $1.351 E-3$ \\
\hline$\varepsilon=2^{-18}$ & $\begin{array}{c}2.546 E-2 \\
1.289\end{array}$ & $\begin{array}{c}1.042 E-2 \\
1.383\end{array}$ & $\begin{array}{c}3.995 E-3 \\
1.561\end{array}$ & $1.354 E-3$ \\
\hline$\varepsilon=2^{-20}$ & $\begin{array}{c}2.551 E-2 \\
1.290\end{array}$ & $\begin{array}{c}1.043 E-2 \\
1.383\end{array}$ & $\begin{array}{c}4.000 E-3 \\
1.562\end{array}$ & $1.355 E-3$ \\
\hline$\varepsilon=2^{-22}$ & $\begin{array}{c}2.553 E-2 \\
1.291\end{array}$ & $\begin{array}{c}1.044 E-2 \\
1.383\end{array}$ & $\begin{array}{c}4.003 E-3 \\
1.562\end{array}$ & $1.356 E-3$ \\
\hline$\varepsilon=2^{-24}$ & $\begin{array}{c}2.555 E-2 \\
1.291\end{array}$ & $\begin{array}{c}1.044 E-2 \\
1.383\end{array}$ & $\begin{array}{c}4.004 E-3 \\
1.562\end{array}$ & $1.356 E-3$ \\
\hline$\varepsilon=2^{-26}$ & $\begin{array}{c}2.555 E-2 \\
1.291\end{array}$ & $\begin{array}{c}1.044 E-2 \\
1.383\end{array}$ & $\begin{array}{c}4.005 E-3 \\
1.562\end{array}$ & $1.356 E-3$ \\
\hline $\begin{array}{l}D^{N} \\
p_{\text {uni }}^{N}\end{array}$ & $\begin{array}{c}2.555 E-2 \\
1.291\end{array}$ & $\begin{array}{c}1.044 E-2 \\
1.383\end{array}$ & $\begin{array}{c}4.005 E-3 \\
1.562\end{array}$ & $1.356 E-3$ \\
\hline
\end{tabular}

TABLE 2. Values of $C_{p^{*}}^{N}$ for problem (4.1)

\begin{tabular}{|cccc|}
\hline$N=32$ & $N=64$ & $N=128$ & $N=256$ \\
\hline $\mathbf{3 . 7 9 1}$ & 3.790 & 3.558 & 2.948 \\
\hline
\end{tabular}

Finally, following [3], we estimate the $\varepsilon$-uniform error constant. From the value

$$
p^{*}=\min _{N} p_{\text {uni }}^{N},
$$

we calculate (see Table 2)

$$
C_{p^{*}}^{N}=\frac{D^{N} N^{p^{*}}}{1-2^{-p^{*}}}
$$

Then, the $\varepsilon$-uniform error constant is defined by

$$
C_{p^{*}}^{*}=\max _{N} C_{p^{*}}^{N}
$$




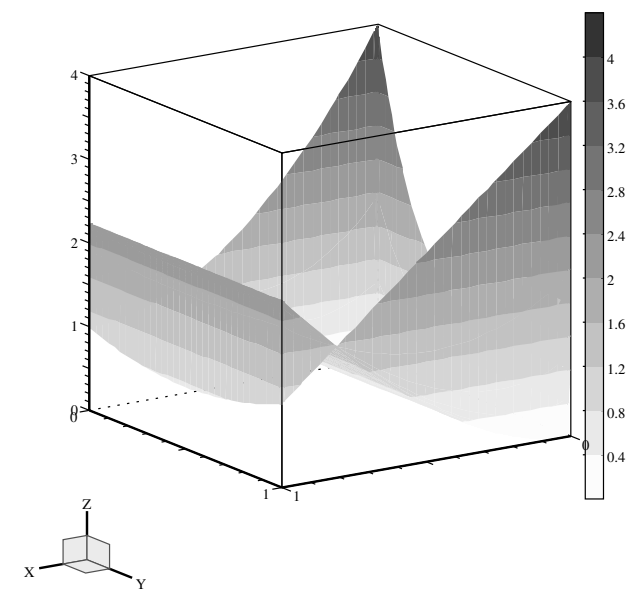

Figure 3. Numerical solution of problem (4.2) $\left(\varepsilon=10^{-6}, N=64\right)$

which in this case takes the value 3.791. As in 3, we propose the following parameter-uniform error estimate for the numerical approximations $U^{N}$ of (3.1) to the solution $u$ of problem (1.1)

$$
\left\|u-U^{N}\right\| \leq 3.79 N^{-1.29}, \quad N \geq 32 .
$$

The second test problem we consider is

$$
\begin{aligned}
& -\varepsilon \Delta u+(1+x)^{2} u=\left(x^{2}+2 x\right)^{2}, \quad(x, y) \in(0,1)^{2}, \\
& u(x, 0)=1+3(1-x)^{2}, \quad 0 \leq x \leq 1, \\
& u(0, y)=4(1-2 y(1-y))^{2}, \quad 0 \leq y \leq 1, \\
& u(x, 1)=1+3(1-x), \quad 0 \leq x \leq 1, \\
& u(1, y)=1-y(1-y), \quad 0 \leq y \leq 1 .
\end{aligned}
$$

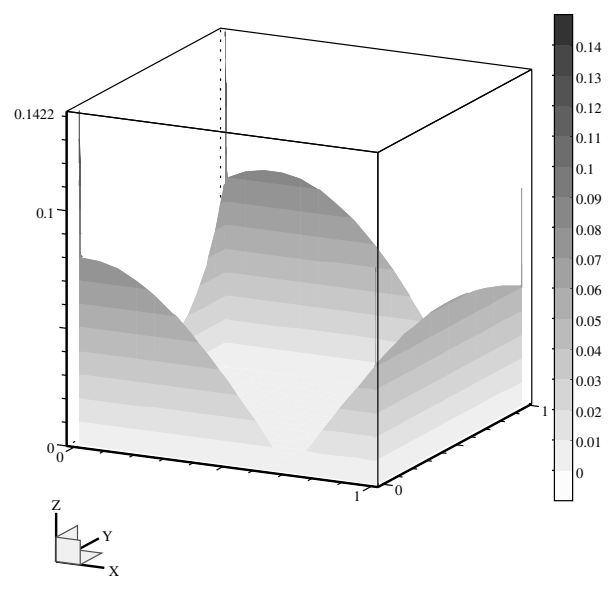

Figure 4. Approximate errors $\left\|U^{32}-\tilde{U}^{512}\right\|$ for problem (4.2) $\left(\varepsilon=10^{-6}\right)$ 
TABLE 3. Maximum differences $D_{\varepsilon}^{N}$ and the numerical orders of convergence $p_{\varepsilon}^{N}$ for problem (4.2)

\begin{tabular}{|c|c|c|c|c|}
\hline$\varepsilon$ & $N=32$ & $N=64$ & $N=128$ & $N=256$ \\
\hline$\varepsilon=1$ & $\begin{array}{c}1.445 E-3 \\
1.998\end{array}$ & $\begin{array}{c}3.618 E-4 \\
1.999\end{array}$ & $\begin{array}{c}9.049 E-5 \\
2.000\end{array}$ & $2.263 E-5$ \\
\hline$\varepsilon=2^{-2}$ & $\begin{array}{c}1.069 E-3 \\
1.998\end{array}$ & $\begin{array}{c}2.678 E-4 \\
1.999\end{array}$ & $\begin{array}{c}6.698 E-5 \\
2.000\end{array}$ & $1.675 E-5$ \\
\hline$\varepsilon=2^{-4}$ & $\begin{array}{c}1.765 E-3 \\
1.953\end{array}$ & $\begin{array}{c}4.558 E-4 \\
1.985\end{array}$ & $\begin{array}{c}1.151 E-4 \\
1.995\end{array}$ & $2.888 E-5$ \\
\hline$\varepsilon=2^{-6}$ & $\begin{array}{c}6.291 E-3 \\
1.881\end{array}$ & $\begin{array}{c}1.708 E-3 \\
1.965\end{array}$ & $\begin{array}{c}4.376 E-4 \\
1.990\end{array}$ & $1.102 E-4$ \\
\hline$\varepsilon=2^{-8}$ & $\begin{array}{c}2.209 E-2 \\
1.780\end{array}$ & $\begin{array}{c}6.430 E-3 \\
1.899\end{array}$ & $\begin{array}{c}1.724 E-3 \\
1.973\end{array}$ & $4.393 E-4$ \\
\hline$\varepsilon=2^{-10}$ & $\begin{array}{c}6.813 E-2 \\
1.599 \\
\end{array}$ & $\begin{array}{c}2.249 E-2 \\
1.783\end{array}$ & $\begin{array}{c}6.536 E-3 \\
1.908 \\
\end{array}$ & $1.741 E-3$ \\
\hline$\varepsilon=2^{-12}$ & $\begin{array}{c}9.762 E-2 \\
1.262\end{array}$ & $\begin{array}{c}4.070 E-2 \\
1.234\end{array}$ & $\begin{array}{c}1.731 E-2 \\
1.444\end{array}$ & $6.363 E-3$ \\
\hline$\varepsilon=2^{-14}$ & $\begin{array}{c}9.993 E-2 \\
1.276\end{array}$ & $\begin{array}{c}4.126 E-2 \\
1.247\end{array}$ & $\begin{array}{c}1.739 E-2 \\
1.443\end{array}$ & $6.394 E-3$ \\
\hline$\varepsilon=2^{-16}$ & $\begin{array}{c}1.011 E-1 \\
1.284\end{array}$ & $\begin{array}{c}4.152 E-2 \\
1.253\end{array}$ & $\begin{array}{c}1.743 E-2 \\
1.443 \\
\end{array}$ & $6.410 E-3$ \\
\hline$\varepsilon=2^{-18}$ & $\begin{array}{c}1.017 E-1 \\
1.287\end{array}$ & $\begin{array}{c}4.165 E-2 \\
1.255\end{array}$ & $\begin{array}{c}1.745 E-2 \\
1.443\end{array}$ & $6.418 E-3$ \\
\hline$\varepsilon=2^{-20}$ & $\begin{array}{c}1.020 E-1 \\
1.289\end{array}$ & $\begin{array}{c}4.171 E-2 \\
1.257\end{array}$ & $\begin{array}{c}1.746 E-2 \\
1.443\end{array}$ & $6.422 E-3$ \\
\hline$\varepsilon=2^{-22}$ & $\begin{array}{c}1.021 E-1 \\
1.290\end{array}$ & $\begin{array}{c}4.175 E-2 \\
1.257\end{array}$ & $\begin{array}{c}1.746 E-2 \\
1.443\end{array}$ & $6.424 E-3$ \\
\hline$\varepsilon=2^{-24}$ & $\begin{array}{c}1.022 E-1 \\
1.291\end{array}$ & $\begin{array}{c}4.176 E-2 \\
1.258\end{array}$ & $\begin{array}{c}1.747 E-2 \\
1.443\end{array}$ & $6.425 E-3$ \\
\hline$\varepsilon=2^{-26}$ & $\begin{array}{c}1.022 E-1 \\
1.291\end{array}$ & $\begin{array}{c}4.177 E-2 \\
1.258\end{array}$ & $\begin{array}{c}1.747 E-2 \\
1.443\end{array}$ & $6.426 E-3$ \\
\hline $\begin{array}{l}D^{N} \\
p_{\text {uni }}^{N} \\
\end{array}$ & $\begin{array}{c}1.022 E-1 \\
1.291\end{array}$ & $\begin{array}{c}4.177 E-2 \\
1.258\end{array}$ & $\begin{array}{c}1.747 E-2 \\
1.443\end{array}$ & $6.426 E-3$ \\
\hline
\end{tabular}

TABLE 4. Values of $C_{p^{*}}^{N}$ for problem (4.2)

\begin{tabular}{|cccc|}
\hline$N=32$ & $N=64$ & $N=128$ & $N=256$ \\
\hline $\mathbf{1 3 . 7 4 4}$ & 13.434 & 13.438 & 11.822 \\
\hline
\end{tabular}

Now the solution has four boundary and corner layers as is shown in Figure 3, Note that the reduced solution is not identically zero and that $\|u\|=4$.

Table 3 displays the differences and the order in this case for the same values of $\varepsilon$ as in the previous example. Again we only have zero-order compatibility conditions; nevertheless we observe orders of convergence tending toward two.

Figure 4 shows the pointwise errors for $\varepsilon=10^{-6}$ using the same technique as before. Again we see that the maximum error occurs in the layer regions.

Finally, Table 4 displays the values of $C_{p^{*}}^{N}$ and in bold we indicate the $\varepsilon$-uniform error constant for problem (4.2). 


\section{REFERENCES}

1. T. Apel and G. Lube, Anisotropic mesh refinement for singularly perturbed reaction diffusion problems, Appl. Numer. Math. 26 (1998), 415-433. MR1612364 (99j:65192)

2. V. F. Butuzov, The asymptotic properties of solutions of the equation $\mu^{2} \triangle u-k^{2}(x, y) u=$ $f(x, y)$ in a rectangle, Differential'nye Uravneniya 9 (1973), 1274-1279. MR0340781 (49:5531)

3. P.A. Farrell, A.F. Hegarty, J.J.H. Miller, E. O'Riordan, and G.I. Shishkin, Robust Computational Techniques for Boundary Layers, Chapman and Hall/CRC Press, Boca Raton, U.S.A., 2000. MR.1750671 (2001c:65003)

4. H. Han and R.B. Kellogg, Differentiability properties of solutions of the equation $-\varepsilon^{2} \triangle u+$ $r u=f(x, y)$ in a square, SIAM J. Math. Anal. 21 (1990), 394-408. MR1038899 (91e:35025)

5. O.A. Ladyzhenskaya and N. N. Ural'tseva, Linear and Quasilinear Elliptic Equations, Academic Press, New York and London, 1968. MR0244627 (39:5941)

6. J. Li and I. M. Navon, Uniformly convergent finite element methods for singularly elliptic boundary value problems I: reaction-diffusion type, Comp. Math. Appl. 35 (1998), 57-70. MR:1605555 (98m:65193)

7. T. Linß, Layer-adapted meshes for convection-diffusion problems, Comput. Methods Appl. Mech. Engrg. 192 (2003), 1061-1105. MR1960975 (2004a:65166)

8. J. J. H. Miller, E. O'Riordan, and G. I. Shishkin, Fitted numerical methods for singular perturbation problems, World-Scientific, (Singapore), 1996. MR.1439750 (98c:65002)

9. J.J.H. Miller, E. O'Riordan, G.I. Shishkin, and L.P. Shishkina, Fitted mesh methods for problems with parabolic boundary layers, Proc. Roy. Irish Acad. Sect. A 98A, (1998), 173190. MR1759430 (2001e:65126)

10. E. O'Riordan M. and Stynes, A uniformly accurate finite-element method for a singularly perturbed one-dimensional reaction-diffusion problem, Math. Comp. 47 (1986), 555-570. MR 0856702 (88f:65126)

11. H.-G. Roos, M. Stynes, and L. Tobiska, Numerical Methods for Singularly Perturbed Differential Equations. Convection-Diffusion and Flow Problems, Springer-Verlag, New York, 1996. MR 1477665 (99a:65134)

12. A. H. Schatz and L. B. Wahlbin, On the finite element method for singularly perturbed reaction-diffusion problems in two and one dimensions, Math. Comp. 40 (1983), 47-89. MR0679434 (84c:65137)

13. G.I. Shishkin, Discrete approximation of singularly perturbed elliptic and parabolic equations, Russian Academy of Sciences, Ural section, Ekaterinburg, 1992. (In Russian)

14. M. Stynes and E. O'Riordan, A uniformly convergent Galerkin method on a Shishkin mesh for a convection-diffusion problem, J. Math. Anal. Appl. 214 (1997), 36-54. MR1645503 (99f:65177)

15. G. Sun and M. Stynes, Finite-element methods for singularly high-order elliptic two-point boundary value problems I: reaction-diffusion-type problems, IMA J. Numer. Anal. 15 (1995), 117-139. MR.1311341 (95k:65075)

16. E. A. Volkov, On the differential properties of solutions of boundary value problems for the Laplace and Poisson equations on a rectangle, Trudy Mat. Inst. Syeklov 77 (1965), 89-112. MR 0192077 (33:304)

17. C. Xenophontos and S. R. Fulton, Uniform approximation of singularly perturbed reactiondiffusion problems by the finite element method on a Shishkin mesh, Numer. Methods Partial Differential Eq. 19 (2003), 89-111. MR.1946805(2004a:65159)

Departamento de Matemática Aplicada, Universidad de Zaragoza, Zaragoza, Spain

E-mail address: clavero@unizar.es

Departamento de Matemática Aplicada, Universidad de Zaragoza, Teruel, Spain

E-mail address: jlgracia@unizar.es

School of Mathematical Sciences, Dublin City University, Glasnevin, Dublin 9, IreLAND

E-mail address: eugene.oriordan@dcu.ie 